\title{
Nuclear matter superfluidity in an effective hadronic field model with excluded volume corrections
}

\author{
R. M. Aguirre \\ Departamento de Física, Facultad de Ciencias Exactas, \\ Universidad Nacional de La Plata. \\ C. C. 67 (1900) La Plata, Argentina \\ and IFLP, CCT La Plata, CONICET.
}

\begin{abstract}
Properties of the ${ }^{1} S_{0}$ superfluid phase are studied for symmetric nuclear matter at finite temperature. It is described within a covariant hadronic field model, of the $\sigma-\omega$ type, with addition of density dependent correlations simulating effects due to finite extension of nucleons. The model is solved in a selfconsistent Hartree-Bogoliubov approach, assuming instantaneous interactions in the superfluid phase. A comparison with the results obtained from several hadronic field models is made. Main characteristics of our description of the superfluid gap are in qualitative agreement with some studies using microscopic potentials, although further refinements could improve its performance.
\end{abstract}

\section{Introduction}

Superfluid states in the nuclear environment have been extensively studied as they have a significative role in several physical processes, such as the structure of nuclei out the stability valley and the cooling dynamics of proto-neutron stars.

A variety of models and approximations have been used for this purpose [1, 2, 3, 4, 5, 6, 7, 8, 9, 10, 11, 12, 13, 14, 15, 16, mainly non-relativistic potentials or effective forces, such as the density-dependent Skyrme or Gogny ones. It is not unusual a mixed treatment that combines both schemes in order to simplify involved calculations [1, 2].

Approximately two decades ago a covariant model of the field theory of hadrons, generally known as Quantum Hadro-Dynamics (QHD) 17, 18, was used for the first time to study nuclear matter superfluidity [9]. There are several reasons to use this theoretical framework to deal with nuclear superfluidity, in first place there are practical reasons, some self-consistent calculations are more simply 
stated and easily solved. This property gave rise to a version of QHD, known as Density Dependent Hadron Field Theory [19] which casts Dirac-Brueckner outputs in the QHD language. In second place one must consider field theory as a more adequate tool to make contact with the fundamental theory of strong interactions and the fact that covariant formulations are desirable for astrophysical applications, among others conceptual reasons. Furthermore, the formalism has the versatility to include vacuum effects and finite renormalizations in a coherent way [14, 15].

Since QHD models are formulated as a many-body theory, one of its basic premises is the reproduction of the nuclear matter saturation properties. This can be fulfilled with a few adjustable parameters and even at the lowest order of approximation. Once the free-parameters have been fixed, the QHD model has a noticeable prediction power.

Although a wide spreading of numerical results for the superfluid phase in infinite nuclear matter can be found in the literature, there is a qualitative agreement that the superfluid gap should not exceed $3 \mathrm{MeV}$, and it should vanish for densities around the saturation density [13. Unfortunately, the findings of 9] for superfluid nuclear matter in the ${ }^{1} S_{0}$ phase do not agree with these expectations. A maximum $\Delta_{\max }\left(p_{F}\right) \approx 10 \mathrm{MeV}$ was found there, and only an unphysical reduction of $15 \%$ in the omega-meson mass yield results comparable with currently accepted values.

This situation has not been changed substantially after the evaluation of different corrections into the original scheme. However, the good properties of this treatment have motivated mixed descriptions combining QHD models and conventional potentials [1, 2, 3].

More recently it was claimed that a coherent inclusion of meson proper selfenergy [10, or adjustable quenching factor [11, could bring numerical calculations to the likely values. Further studies about the effect of corrections of the meson propagators and the influence of low density instabilities on the superfluid gap can be found for instance in 12 .

It was stressed in reference 9] that the high momenta behavior of the repulsive potential has a crucial role in the exceedingly large values obtained for $\Delta_{\max }\left(p_{F}\right)$. It must be noticed that the relative strength of repulsive and attractive contributions have been calibrated for momenta below the Fermi surface in order to produce the saturation mechanism. Therefore it would be desirable a pairing potential which preserves the relative strength of its components in the Fermi sphere, but having a repulsive component decreasing as faster as the attractive one in the high momenta domain.

Taking these facts into account we try in the present work, to obtain an effective and concise model able to deal with the superfluid phase of nuclear matter. For this purpose we use and compare several models of relativistic nuclear fields interacting through scalar and vector mesons. As a first approach, we reduce to its minimal expression the complexity of the nuclear interaction, but further refinements can be considered. We introduce a characteristic length scale in the effective interaction, which could eventually be traced back to the confining 
mechanism of the fundamental theory of strong interactions. A similar approach was applied in the past to describe heavy ion collisions [20, 21, 22, 23, 24] as well as nuclear matter properties [25, 26, 27, 28, 29, 30]. It has been known as finite volume correction since it takes into account the spread of the nucleon localization. The relevance of this effect upon the evaluation of some bulk properties of the nuclear matter has been stressed long time ago 31.

In the next section we present the theoretical deduction of the general gap equation at finite temperature in a context of Landau-Fermi liquid. The last part of this section is devoted to the theoretical deduction of the self-consistent expression defining the superfluid gap for the ${ }^{1} S_{0}$ phase within the QHD framework. In section 3 we show the numerical results and discuss them. Finally, conclusions are drawn in section 4 .

\section{The Formalism}

\subsection{Superfluid states in a Landau-Fermi liquid}

The different superfluid phases in a fermionic system can be described in a general and compact way within the formalism of the Fermi liquid, using for instance the formalism of reference [32. It is assumed that the low-lying excitations of the system are represented in terms of quasi-particles and, circumstantially, collective modes.

We use the notation $f_{\alpha}$ for the equilibrium distribution function of a quasiparticle state, where the label $\alpha$ comprise spin, isospin, and momentum quantum numbers. The fermionic contribution to every conserved quantity, such as particle number and energy, can be expressed in terms of a summation over $f_{\alpha}$. In the following we will be interested in nucleon pairs coupled to singlet spin and triplet isospin, so that an anomalous distribution function $g_{\beta}$ and a energy gap $\Delta_{\beta}$ are introduced. According to $\underline{32}$, we make the decomposition

$$
\begin{aligned}
g_{\beta} & =g_{k}(p)\left[\tau^{(k)} \tau^{(2)}\right]_{a b} \sigma_{s s^{\prime}}^{(2)} \\
\Delta_{\beta} & =\Delta_{k}(p)\left[\tau^{(k)} \tau^{(2)}\right]_{a b} \sigma_{s s^{\prime}}^{(2)}
\end{aligned}
$$

where momentum, isospin $(a, b)$, and spin $\left(s, s^{\prime}\right)$ dependencies has been clearly distinguished. Here $\tau_{k}, \sigma_{k}, k=1,2,3$ stands for the Pauli matrices for isospin and spin, respectively.

On the other hand, the normal phase is filled with quasi-particle states in a isospin duplet described similarly by $f_{\beta}=\delta_{s s^{\prime}} f_{a b}(p)$, with

$$
f_{a b}(p)=f_{0}(p) \delta_{a b}+f_{3}(p) \tau_{a b}^{(3)} .
$$

Accordingly, the quasi-particle spectra is assumed in matrix form $\varepsilon(p)=\varepsilon_{0}(p)+$ $\varepsilon_{3}(p) \tau_{3}$. 
In the Landau theory of Fermi liquids, the energy of the system $E$ is considered as a functional of the distribution functions $f_{\alpha}, g_{\beta}$. First variations respect to them give the quasi-particle and gap spectra matrix components

$$
\varepsilon_{j k}(p)=\frac{\delta E}{\delta f_{j k}(p)}, \quad \Delta_{j k}(p)=\frac{\delta E}{\delta g_{j k}^{\dagger}(p)} .
$$

Since $f$ and $g$ are itself functions of $\varepsilon, \Delta$, the equations above are selfconsistent relations.

Within the block diagonalization procedure of 32 the distribution functions are written

$$
\begin{aligned}
f & =K n+X\left(1-n^{t}\right) X^{\dagger} K, \\
g & =(K n X)^{t}+K(1-n) X, \\
n & =\left[1+e^{\beta\left(\xi-X \Delta^{\dagger}\right)}\right]^{-1}, \\
K & =\left(1+X X^{\dagger}\right)^{-1},
\end{aligned}
$$

here $t$ indicates matrix transposition, $\beta=1 / k T, \xi=\varepsilon+\mu$, and the diagonal ma$\operatorname{trix} \mu=\operatorname{diag}\left(\mu_{1}, \mu_{2}\right)$ collects the proton (1) and nucleon (2) chemical potentials. The unknown matrix $X=X_{j} \tau_{j} \tau_{2} \sigma_{2}$ satisfies the condition

$$
\xi X+X \xi^{T}+\Delta-X \Delta^{\dagger} X=0
$$

We have solved this system of equations for symmetric nuclear matter coupled to $T_{z}=0$. We obtained

$$
\begin{aligned}
& X_{i}=\Delta_{i}=g_{i}=0, \text { for } i=1,2, X_{3}=\frac{\varepsilon_{0}-\mu_{0} \pm E_{\Delta}}{\Delta_{3}^{*}} \\
& g_{3}(p)=-\frac{\Delta_{3}}{2 E_{\Delta}} \tanh \left(\beta E_{\Delta} / 2\right), f_{0}=\frac{1}{2}\left[1-\frac{\varepsilon_{0}-\mu_{0}}{E_{\Delta}} \tanh \left(\beta E_{\Delta} / 2\right)\right], \\
& f_{3}=0, E_{\Delta}=\sqrt{\Delta_{3}^{2}+\left(\varepsilon_{0}-\mu_{0}\right)^{2}}
\end{aligned}
$$

in the first line $\mu_{0}$ stands for either the proton or the neutron chemical potential. The particle density of protons $(\mathrm{k}=1)$ or neutrons $(\mathrm{k}=2)$ is given by

$$
n_{k}=\int \frac{d^{3} p}{(2 \pi)^{3}}\left[f_{0}(p)+\tau_{k k}^{(3)} f_{3}(p)\right]
$$

these equations are used to relate the chemical potentials to the conserved isospin and baryonic number density.

In the next subsection we show the model which provides the quasi-particle interaction and spectra.

\subsection{Hadronic models}

Models of the nuclear interaction, formulated in the covariant field theory, have been widely used in the study of the dynamics and structure of infinite matter 
as well as finite nuclei. Since the pioneering work of references [17, 18, the simple $\sigma-\omega$ model has grown in different directions and it was completed in order to cover a multitude of manifestations of the nuclear force.

In particular the subject of the nuclear superfluidity was first treated within this context in reference [9], by using the original $\sigma-\omega$ model plus a pseudoscalar pion interaction. The scheme of approximation used there consisted in a mean field treatment of the meson and nucleon fields, a Gorkov factorization of the pairing interaction and a instantaneous assumption which allows a timeindependent resolution of the gap equation. Subsequently, this procedure was extended to consider the effect of vacuum, and the variation of the in-medium meson properties [14, 15.

Most of these studies agree in a excessively large value for the gap in infinite nuclear matter. The realization of a correlated state of two nucleons is a consequence of the equilibrium between a repulsive and an attractive component of the pairing potential, originating in the exchange of virtual $\omega$-mesons and $\sigma$-mesons respectively. This mechanism is also found in the binding energy of nuclear matter.

The slow decrease of the repulsive potential as a function of the transferred momenta, has been pointed out as the main cause of the mismatch. This situation can not be modified without a substantial redefinition of the couplings, which should lead to a destruction of the saturation mechanism.

In this work we adopt the simplest version of nucleons interacting through $\sigma$ and $\omega$ mesons, whose lagrangian density is

$\mathcal{L}=\bar{\Psi}^{a}\left(i \not \partial-M_{a}+g_{s} \sigma-g_{w} \not\right) \Psi^{a}+\frac{1}{2}\left(\partial^{\mu} \sigma \partial_{\mu} \sigma-m_{s}^{2} \sigma^{2}\right)-\frac{1}{4} F^{\mu \nu} F_{\mu \nu}+\frac{1}{2} m_{w}^{2} \omega^{2}$

where summation over the repeated isospin index $a$ is assumed, $F_{\mu \nu}=\partial_{\mu} \omega_{\nu}-$ $\partial_{\nu} \omega_{\mu}$ and $g_{s}, g_{w}$ are adimensional coupling constants. The equations of motion of the classical fields are

$$
\begin{aligned}
\left(i \not \partial-M_{a}+g_{s} \sigma-g_{w} \not\right) \Psi^{a} & =0 \\
\left(\square+m_{s}^{2}\right) \sigma-g_{s} \bar{\Psi}^{a} \Psi^{a} & =0 \\
\partial_{\nu} F_{\mu}^{\nu}+m_{w}^{2} \omega_{\mu}-g_{w} \bar{\Psi}^{a} \gamma_{\mu} \Psi^{a} & =0
\end{aligned}
$$

Denoting by $D(x, y), D_{\mu \nu}(x, y)$ the propagators of scalar and vector-meson fields, the eqs. (9) and (10) can be formally solved as

$$
\begin{aligned}
\sigma(x) & =g_{s} \int d^{4} y D(x, y) \Psi^{a}(y) \Psi^{a}(y) \\
\omega_{\mu}(x) & =g_{w} \int d^{4} y D_{\mu \nu}(x, y) \Psi^{a}(y) \gamma^{\nu} \Psi^{a}(y)
\end{aligned}
$$

The Hamiltonian density $\mathcal{H}$ is given by the canonical procedure, and the energy density of the system $\mathcal{E}$ is evaluated by taking its expectation value 
$\mathcal{E}=<\bar{\Psi}^{a} i \gamma_{0} \partial_{0} \Psi^{a}+\partial_{0} \sigma \partial_{0} \sigma-F_{0 \mu} \partial_{0} \omega^{\mu}+\frac{1}{2} \sigma\left(\square+m_{s}^{2}\right) \sigma-\frac{1}{2} \omega_{\nu}\left(\partial_{\mu} F^{\mu \nu}+m_{w}^{2} \omega^{\nu}\right)>$

Under the hypothesis of static meson fields the second and third terms vanish.

Inserting the meson equations of motion (91), (10) together with the Eq. (12) into (13), we obtain

$$
\begin{aligned}
\mathcal{E}(x)= & <\bar{\Psi}^{a}(x) i \gamma_{0} \partial_{0} \Psi^{a}(x)>+\frac{g_{s}^{2}}{2} \int d^{4} y D(x, y)<\Psi_{\alpha}^{a}(x) \Psi_{\alpha}^{a}(x) \Psi_{\beta}^{b}(y) \Psi_{\beta}^{b}(y)> \\
& \left.-\frac{g_{w}^{2}}{2} \int d^{4} y D_{\mu \nu}(x, y) \gamma_{\alpha \alpha^{\prime}}^{\mu} \gamma_{\beta \beta^{\prime}}^{\nu}\right)<\Psi_{\alpha}^{a}(x) \Psi_{\alpha^{\prime}}^{a}(x) \Psi_{\beta}^{b}(y) \Psi_{\beta^{\prime}}^{b}(y)>
\end{aligned}
$$

At this point we introduce an expansion of the nucleon fields in terms of quasi-particle creation and annihilation operators, similar to that of a free field

$$
\begin{aligned}
\Psi_{\alpha}^{a}(x) & =\int d^{3} p \frac{M_{a}^{*}}{E_{a}(p)}\left[b_{s}^{a}(p) u_{\alpha}^{s}(p) e^{-i P_{a} x}+d_{s}^{\dagger a}(p) v_{\alpha}^{s}(p) e^{i P_{a} x}\right] \\
\bar{\Psi}_{\alpha}^{a}(x) & =\int d^{3} p \frac{M_{a}^{*}}{E_{a}(p)}\left[b_{s}^{\dagger a}(p) \bar{u}_{\alpha}^{s}(p) e^{i P_{a} x}+d_{s}^{a}(p) \bar{v}_{\alpha}^{s}(p) e^{-i P_{a} x}\right] \\
u_{a}^{s}(p) & =\frac{P_{a}+M_{a}^{*}}{\sqrt{2 M_{a}^{*}\left(M_{a}^{*}+E_{a}(p)\right)}} u^{s}(0), \\
v_{a}^{s}(p) & =\frac{P_{a}+M_{a}^{*}}{\sqrt{2 M_{a}^{*}\left(M_{a}^{*}+E_{a}(p)\right)}} u^{s}(0),
\end{aligned}
$$

but creation and annihilation operators are referred to the lowest energy state of correlated nucleons. We have used the quasi-particle properties $M_{a}^{*}=M-g_{s} s$, $E_{a}(p)=\sqrt{M_{a}^{* 2}+p^{2}}, P_{a}=\left(E_{a}(p)+g_{w} w, \mathbf{p}\right)$. Due to the isospin invariance of the interaction proton and neutron properties are actually independent. Furthermore, as we are interested in isospin symmetric matter proton and neutron are indistinguishable and the isospin index $a$ becomes superfluous. From now on we will omit it, and a degeneracy factor 2 will be included when necessary. The quantities $s, w$ stand for the mean values of the $\sigma$ and $\omega$ meson fields in homogeneous, isotropic matter. As usual, they can be deduced from Eqs. (9), (10) by neglecting derivatives and taking expectation values of the fermionic bilinears $s=2 g_{s}<\bar{\Psi} \Psi>/ m_{s}^{2}, w=2 g_{w}<\bar{\Psi} \gamma_{0} \Psi>/ m_{w}^{2}$.

As a part of the approximation we neglect in Eq. (14) the contribution of particle-antiparticle or antiparticle-antiparticle terms. We define the equilibrium distribution functions for the normal and superfluid phases

$$
\begin{aligned}
f_{s s^{\prime}}(p, k) & =\frac{M^{*}}{\sqrt{E(p) E(k)}}<b_{s^{\prime}}^{\dagger}(k) b_{s}(p)>, \\
g_{s s^{\prime}}(p, k) & =\frac{M^{*}}{\sqrt{E(p) E(k)}}<b_{s^{\prime}}(k) b_{s}(p)>, \\
g_{s s^{\prime}}^{\dagger}(p, k) & =\frac{M^{*}}{\sqrt{E(p) E(k)}}<b_{s^{\prime}}^{\dagger}(k) b_{s}^{\dagger}(p)>.
\end{aligned}
$$


It must been taken into account that $f(k, p) \propto(2 \pi)^{3} \delta^{3}(p-k)$, whereas pairs of nucleons are assumed to couple to zero momentum, so that $g(k, p) \propto(2 \pi)^{3} \delta^{3}(p+$ $k)$.

Within the mean field approach, corrections to the meson propagation in the nuclear environment are dismissed, although a random phase approximation could be considered, as in [10]. In the first case, we obtain

$$
\begin{aligned}
D(z) & =\int d^{4} q D(q) e^{-i q z} \\
D(q) & =-\left(q_{\lambda} q^{\lambda}-m_{s}^{2}+i \varepsilon\right)^{-1}, \\
D_{\mu \nu}(q) & =-\left(g_{\mu \nu}-q_{\mu} q_{\nu} / m_{w}^{2}\right) /\left(q_{\lambda} q^{\lambda}-m_{w}^{2}+i \varepsilon\right),
\end{aligned}
$$

The term proportional to $q_{\mu}$ in (22), produce zero contribution in integrals combining $D_{\mu \nu}$ with nucleon fields, because of the conservation of the baryonic current.

We are interested in static homogeneous matter, therefore $z=x-x^{\prime}$ in Eq. (20). If we neglect time retardation in the meson propagation, $i$. e. $0=x_{0}-x_{0}^{\prime}$, then terms containing $q_{0}$ are absent in Eqs. (21), (22) as well as in the exponential of the Fourier transform shown lines above.

All these elements together in Eq. (14) produce

$$
\begin{aligned}
\mathcal{E}= & \mathcal{E}_{M F A}+\mathcal{E}_{F}+\mathcal{E}_{S} \\
\mathcal{E}_{M F A}= & 4 \int d^{3} p \varepsilon(p) f(p)+2\left(\frac{2 g_{s}}{m_{s}} \int d^{3} p \frac{M^{*}}{E(p)} f(p)\right)^{2} \\
& -2\left(\frac{2 g_{w}}{m_{w}} \int d^{3} p f(p)\right)^{2} \\
\mathcal{E}_{F}= & 2 g_{s}^{2} \int d^{3} p d^{3} q \frac{f(p) f(q)}{2 E(p) E(q)} \frac{M^{* 2}+E(p) E(q)-\mathbf{p} \cdot \mathbf{q}}{[\varepsilon(p)-\varepsilon(q)]^{2}-|\mathbf{p}-\mathbf{q}|^{2}-m_{s}^{2}+i \varepsilon} \\
& -2 g_{w}^{2} \int d^{3} p d^{3} q \frac{f(p) f(q)}{E(p) E(q)} \frac{2 M^{* 2}-E(p) E(q)+\mathbf{p} \cdot \mathbf{q}}{[\varepsilon(p)-\varepsilon(q)]^{2}-|\mathbf{p}-\mathbf{q}|^{2}-m_{w}^{2}+i \varepsilon} \\
\mathcal{E}_{S}= & -g_{s}^{2} \int d^{3} p d^{3} q \frac{g^{\dagger}(p) g(q) \Lambda_{s}(p, q)}{[\varepsilon(p)-\varepsilon(q)]^{2}-|\mathbf{p}-\mathbf{q}|^{2}-m_{s}^{2}+i \varepsilon} \eta(p, q) \\
& +g_{w}^{2} \int d^{3} p d^{3} q \frac{g^{\dagger}(p) g(q) \Lambda_{w}(p, q)}{[\varepsilon(p)-\varepsilon(q)]^{2}-|\mathbf{p}-\mathbf{q}|^{2}-m_{s}^{2}+i \varepsilon} \eta(p, q) .
\end{aligned}
$$

Here we have separated mean field $(M F A)$, Fock $(F)$ and superfluid $(S)$ contributions. Certain integrals appearing in (14) vanish because of the isotropy of infinite matter. The following notation is used

$$
\begin{aligned}
\Lambda_{s}(p, q) & =\frac{X^{2}(p, q)-2 X(p, q) \mathbf{p} \cdot \mathbf{q}+p^{2} q^{2}}{E(p) E(q) X(p, q)} \\
\Lambda_{w}(p, q) & =\Lambda_{s}(p, q)+\frac{3 X(p, p) q^{2}+3 X(q, q) p^{2}-2 X(p, q) \mathbf{p} \cdot \mathbf{q}+p^{2} q^{2}}{E(p) E(q) X(p, q)} \\
\eta(p, q) & =\exp \left(-i 2 x_{0}[\varepsilon(q)-\varepsilon(p)]\right)
\end{aligned}
$$


with $X(p, q)=\left(M^{*}+E(p)\right)\left(M^{*}+E(q)\right)$.

The factor $\mathbf{p} \cdot \mathbf{q}=p q \nu$ entering in these expressions can be used for integration respect to $\nu$. For this purpose it is useful the distribution identity

$$
\frac{1}{z+i \varepsilon}=P V\left(\frac{1}{z}\right)-i \pi \delta(z)
$$

Denominators in the integrands of Eqs. (25), (24) come from the meson propagators, in particular the combinations $\varepsilon(q)-\varepsilon(p)$ come from its $q_{0}$ dependence. In the next step we apply the instantaneous approximation [9], which result in the elimination of all these combinations.

Within this approach Eq. (25) can be re-written

$$
\begin{aligned}
\mathcal{E}_{S} & =-g_{s}^{2} \int d^{3} p d^{3} q \frac{g(q) g^{\dagger}(p)}{E(p) E(q)} \\
& +g_{s}^{2} \int d^{3} p d^{3} q g(q) g^{\dagger}(p) \frac{X^{2}(p, q)+p^{2} q^{2}-X(p, q)\left(p^{2}+q^{2}+m_{s}^{2}\right)}{4 p q E(p) E(q) X(p, q)} \ln \left(\frac{(p-q)^{2}+m_{s}^{2}}{(p+q)^{2}+m_{s}^{2}}\right) \\
& -g_{w}^{2} \int d^{3} p d^{3} q g(q) g^{\dagger}(p) \frac{X^{2}(p, q)+p^{2} q^{2}+3 X(p, p) q^{2}+3 X(q, q) p^{2}}{4 p q E(p) E(q) X(p, q)} \ln \left(\frac{(p-q)^{2}+m_{w}^{2}}{(p+q)^{2}+m_{w}^{2}}\right)
\end{aligned}
$$

From now on, we use only the MFA and neglect the Fock term. The superfluid gap can be determined by using Eqs. (2) and (3)

$$
\begin{aligned}
\Delta_{3}(p)= & -\frac{g_{s}^{2}}{8} \int d^{3} q \frac{g_{3}(q)}{E(p) E(q)}+\frac{g_{s}^{2}}{16} \int d^{3} q g_{3}(q) \ln \left(\frac{(p-q)^{2}+m_{s}^{2}}{(p+q)^{2}+m_{s}^{2}}\right) \frac{4 M^{2}-(E(p)-E(q))^{2}-m_{s}^{2}}{p q E(p) E(q)} \\
& -\frac{g_{w}^{2}}{8} \int d^{3} q g_{3}(q) \ln \left(\frac{(p-q)^{2}+m_{w}^{2}}{(p+q)^{2}+m_{w}^{2}}\right) \frac{2 E(p) E(q)-M^{2}}{p q E(p) E(q)}
\end{aligned}
$$

Finally it must be stressed that in applying Eq.(3) for evaluating the quasiparticle spectra, the effective mass $M^{*}$ must be considered a functional of the distribution function $f$.

\subsection{Effects of the spatial extension of nucleons}

Standard field theory considers physical particles as structureless, point-like objects. This could be a serious shortcoming when composed states in a dense medium are described. It is well known that, for instance, the energy and charge density of a soliton spreads over a finite range of space 33. Therefore it is legitimate to assign a intrinsical length scale to nucleons immersed in a dense environment.

This was the argument supporting many phenomenological studies of the nuclear interaction [28, 29, 30, 31]. From a practical point of view, one can consider $N_{a}$ fermions of class $a$ distributed over a finite volume $V$, then the available space for quantization is $V^{\prime}=V-\sum_{a} N^{a} v_{a}$, where $v_{a}$ is the spatial extension of this state. But the canonical procedure uses the full volume $V$, this situation is corrected 
by introducing a correction factor $\sqrt{V^{\prime} / V}$ in the second quantization of fields. This, in turn, modifies fermion bilinears like particle number $n_{a}=\left\langle\bar{\Psi}_{a} \Psi_{a}\right\rangle$ and normal energy density by a factor $\Theta=V^{\prime} / V=1-\sum_{b} n^{b} v_{b}$. The superfluid energy density requires a correction $\Theta^{2}$, which is transferred to the formulae of the gap function.

The effective volume inaccessible for other particles due to the presence of a spherical object of radius $R_{a}$ is

$$
v_{a}=\alpha \frac{4 \pi}{3} R_{a}{ }^{3},
$$

The parameter $R_{a}$ can be understood in a simple minded model as the geometrical size of a particle. Actually it introduces into the model a characteristic scale of the strong interaction, i. e. the spatial spreading of a bounded state of quarks and gluons. The value selected for this radius must be compatible with similar lengths adopted in hybrid models of the nuclear interaction, see for instance 34]. Returning to the schematic picture, the factor $\alpha$ takes account of the fact that the inaccessible volume exceeds the actual size of each particle and it depends on the spatial arrangement adopted by the collection of objects. The minimal volume configuration for identical particles corresponds to a face centered cubic arrangement. In such a case is $\alpha=3 \sqrt{2} / \pi$, which is the value adopted in the present calculations.

It is worthwhile to mention that the normalization of the nucleon field with an excluded volume coefficient is not equivalent to the introduction of a hardcore potential. The normalized nucleon field interacts dynamically with the meson fields, both scalar and vector. The in-medium properties of protons and neutrons, as well as the meson fields configuration arise simultaneously from this interaction. The sigma meson gives rise to the attractive channel of the nuclear force, whereas the omega meson is responsible for the repulsive component. Therefore, the proposed normalization affects both attractive and repulsive channels. This fact is evident from Eq. (9) and the discussion given above. It should be clear that the mean-field value of the sigma meson is strongly affected by the normalization of the nucleons. Moreover, the relation is highly non-linear. Furthermore, the treatment of the mesons is not symmetrical since the omega meson is coupled to a conserved charge. In consequence the omega meson mean field value is completely determined by the conserved baryonic density. The sigma meson mean field value, instead, come forth the hadronic dynamics.

Since $\Theta$ introduces an explicit dependence upon the baryonic densities, the quasi-particle energy $\varepsilon$, see Eq. (3), gets an extra term

$$
\varepsilon_{a}(p)=\sqrt{p^{2}+M_{a}^{* 2}}+g_{w} w+\frac{2 v_{a}}{3} \int d^{3} q \frac{q^{2} f^{a}(q)}{E_{a}(q)},
$$

it must be noticed that the additional term depends on density and temperature, but not on the momentum. 


\begin{tabular}{ccc}
$R[\mathrm{fm}]$ & $\Delta_{\max }^{F E C} / \Delta_{\max }^{N F E C}$ & $g_{w} / g_{s}$ \\
\hline 0.6 & 0.68 & 1.222 \\
0.62 & 0.64 & 1.209 \\
0.64 & 0.61 & 1.193 \\
0.66 & 0.59 & 1.171 \\
0.68 & 0.58 & 1.141 \\
0.70 & 0.56 & 1.095 \\
0.71 & 0.57 & 1.062 \\
0.72 & 0.60 & 1.018
\end{tabular}

Table 1: The gap function on the Fermi surface and the quotient of coupling constants for several values of the characteristic nucleon size $R$.

\section{Results and Discussion}

The model of nucleons and mesons with finite volume corrections has several parameters, we used $M=940 \mathrm{MeV}$ for the mass of the degenerate nucleons, and $m_{w}=783 \mathrm{MeV}$ for the omega-meson mass. The sigma-meson mass has been fixed at $m_{s}=520 \mathrm{MeV}$, in agreement with [9]. A discussion about variation of $m_{s}$ can be found in [9].

The length scale $R_{a}$ is not determined by the model, therefore we consider it as a constant value ranging between $0.5 \mathrm{fm}$ and $0.9 \mathrm{fm}$.

The coupling constants $g_{s}, g_{w}$ are fixed in order to reproduce the saturation properties of symmetric nuclear matter, the binding energy $E_{B}=-16 \mathrm{MeV}$ and a saturation density corresponding to the Fermi momentum $p_{F}=1.42 \mathrm{fm}^{-1}$ [9, 11.

To have a look of the performance of QHD models in describing the nuclear superfluidity, we have examined two different models of the nucleon-meson interaction. In first place we study the density dependent coupling model of [19], which translate Dirac-Bruckner calculations with Bonn A potential into the covariant field theory. We have used the rational function parametrization given there for the couplings, obtaining a maximum value $\Delta_{\max }\left(p_{F}\right) \simeq 15$ $\mathrm{MeV}$ at $n / n_{0}=0.25$. As another QHD example we take the non-linear mesonnucleon model of [35, it predicts a lower compressibility and higher effective mass in the MFA than the $\sigma-\omega$ model [17, 18, does. In our calculations we get $\Delta_{\max }\left(p_{F}\right)=10.6 \mathrm{MeV}$ at $n / n_{0} \simeq 0.1$.

As the next step we consider the effects of the finite extension of nucleons, taking $R$ as an adjustable parameter. The results are summarized in Table 1 , where the treatments regarding the finite extension of nucleons (FEC) or neglecting it (NFEC) have been distinguished. There is a sensible reduction of $\Delta_{\max }$, between $30 \%$ and $40 \%$, respect to the previous calculations. A nonmonotonous dependence on $R$ is obtained, the lowest value $\Delta_{\max }=5.5 \mathrm{MeV}$ is reached for $R=0.7 \mathrm{fm}$ at $p_{F}=0.8 \mathrm{fm}^{-1}$.

Choosing $R=0.7 \mathrm{fm}^{-1}$ for subsequent calculations, we compare the momentum dependence of the gap function for a fixed density $n / n_{0}=0.25$. Results are 
shown in Fig.1. The square points show the magnitude of the gap at the Fermi surface. A comparison of the two cases NFEC and FEC, shows that the latter yields the lowest absolute value for $q<1.6 \mathrm{GeV}$. A reduction of almost $40 \%$ is registered at the Fermi surface in the FEC case.

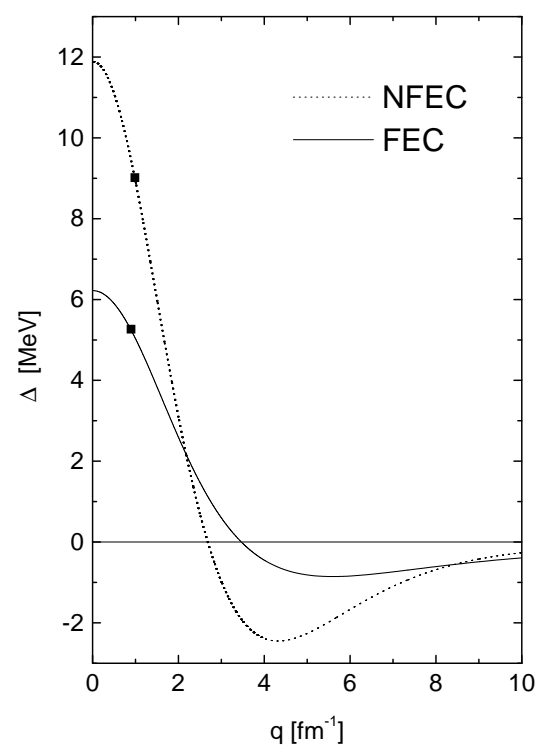

Figure 1: The gap energy as a function of the momentum, square symbols indicate its value at the Fermi surface.

In Fig. 2 we display $\Delta\left(p_{F}\right)$ as a function of the density, here one can see that the FEC treatment not only lowers $\Delta_{\max }$, but also reduces more than $20 \%$ the range of densities where the gap is effective. In this case $p_{F}=1.6 \mathrm{fm}^{-1}$ is the upmost value for non-zero pairing gap.

To improve understanding of this outcome, we investigate separately contributions of scalar and vector character to the gap function. The attractive $\left(v_{s}\right)$ and repulsive $\left(v_{w}\right)$ potentials evaluated at the Fermi surface

$$
\begin{aligned}
& v_{s}(q)=-\frac{g_{s}^{2}}{8 E_{F} E(q)}\left[1+\frac{4 M^{2}-2\left(E_{F}-E(q)\right)^{2}-m_{s}^{2}}{2 p_{F} q} \ln \left(\frac{\left(p_{F}-q\right)^{2}+m_{s}^{2}}{\left(p_{F}+q\right)^{2}+m_{s}^{2}}\right)\right] \\
& v_{w}(q)=-\frac{g_{w}^{2}}{8} \frac{2 E_{F} E(q)-M^{2}}{p_{F} q E_{F} E(q)} \ln \left(\frac{\left(p_{F}-q\right)^{2}+m_{w}^{2}}{\left(p_{F}+q\right)^{2}+m_{w}^{2}}\right)
\end{aligned}
$$

where $E_{F}=\sqrt{p_{F}^{2}+M^{2}}$, have been defined in order that Eq. (26) can be simplified to

$$
\Delta_{3}\left(p_{F}\right)=\int_{0}^{\infty} d q g_{3}(q)\left[v_{s}(q)+v_{w}(q)\right] .
$$

As can be seen in figure 3 both $v_{s}$ and $v_{w}$ appears diminished in the FEC case, with a stronger suppression of $v_{s}$ as compared to $v_{w}$ in the high momenta regime. Therefore $v_{s}+v_{w}$ asymptotically goes to zero faster in FEC than in 


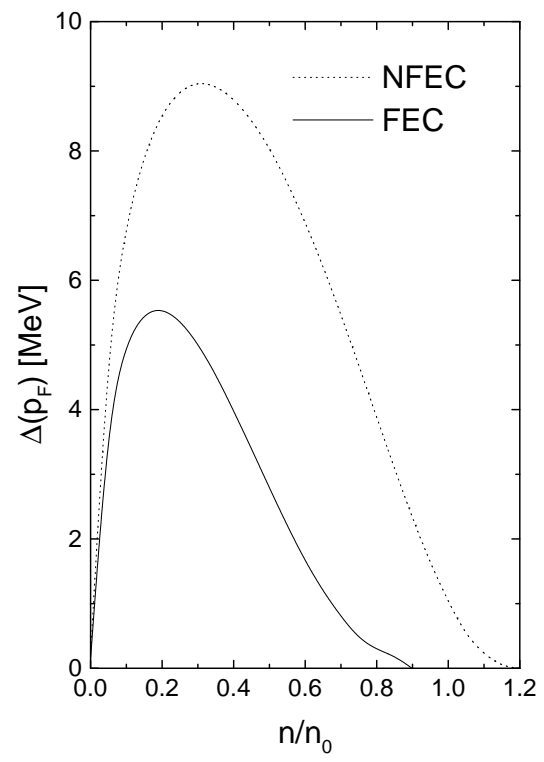

Figure 2: The energy gap evaluated on the Fermi surface in terms of the particle density. Results including Finite Extension Corrections (FEC) or not (NFEC).

NEFC, minimizing contributions from higher momenta.

On the other hand, for $q<1 \mathrm{fm}^{-1}$ the attractive potential is stronger than the repulsive one in FEC than in NFEC approaches.

There is a surprising numerical similitude between the values described above for $\Delta_{\max }\left(p_{F}\right)$ and some findings in [7, where a sophisticated evaluation of in medium effects over the ${ }^{1} S_{0}$ pairing is presented, within the Brueckner theory of nuclear matter. The BCS gap function is found to have a maximum value $\Delta_{\max } \simeq 5 \mathrm{Mev}$ in symmetric nuclear matter at $p_{F}=0.9 \mathrm{fm}^{-1}$. The upper limit for the existence of the gap is given by $p_{F}=1.5 \mathrm{fm}^{-1}$.

Another interesting comparison can be made with the results found by Matsuzaki et al. [16. That work, as the present approach, evaluate the possibility of giving a unified description of both particle-particle and particle-hole channels. In that case the sigma-omega model is used in the mean field approximation, and the results are rendered physically acceptable by a direct intervention over the integrals in momentum space. This is achieved by the introduction of a form factor depending on a single parameter, which is adjusted to obtain the best fit to microscopic calculations. It must be pointed out that the correction factor $\Theta$ used in our procedure can not be rigorously considered as a form factor, since for a given Fermi momentum it reduces to a constant value and therefore it does not modify the integrals. Despite the procedural differences, our results are comparable to those of [16. For instance, if we consider the momentum dependence of the single-particle potential $v\left(p_{F}, q\right)=v_{s}+v_{w}$, evaluated at $p_{F}$ such that a maximum of $\Delta_{\max }\left(p_{F}\right)$ is obtained, it takes values $-4 \mathrm{MeV}<v\left(p_{F}, q\right)<3 \mathrm{MeV}$ in [16. In our calculations, instead, we found -5 


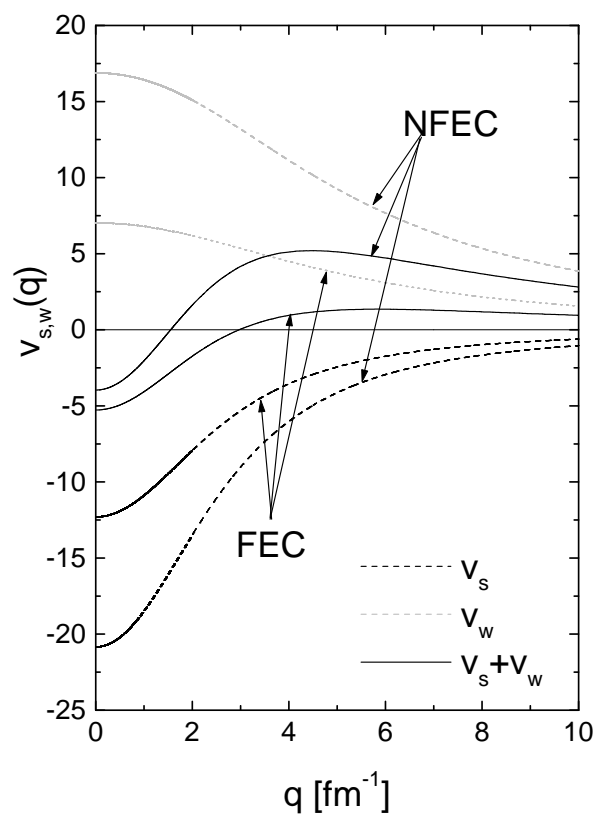

Figure 3: The pairing potential and its repulsive and attractive components in terms of the particle density, for FEC (solid lines) and NFEC (dashed lines) approximations.

$\mathrm{MeV}<v\left(p_{F}, q\right)<1 \mathrm{MeV}$, see Fig. 3. Furthermore, the asymptotic behavior is similar in both calculations. More appreciable differences are found for the momentum dependence of the gap corresponding to the same $p_{F}$. At very low momentum we have $\Delta \sim 6 \mathrm{MeV}$, then as the transfer momentum $q$ is increased, the gap decreases smoothly, passes through zero at $q \sim 3.5 \mathrm{fm}^{-1}$, reaches a minimum value of $-1 \mathrm{MeV}$ at $q \sim 5.5 \mathrm{fm}^{-1}$, and finally tends asymptotically to zero from negative values, see Fig. 1. As can be seen in Fig. 3b of [16, the low momentum gap is sensibly lower $\Delta \sim 4 \mathrm{MeV}$, the gap passes trough zero at a lower value $q \sim 2 \mathrm{fm}^{-1}$ and reaches the same minimum value but at $q \sim 3.5 \mathrm{fm}^{-1}$. From this observations, we can conclude that in our approach, the contributions coming from momenta $q<3 \mathrm{MeV}$ are overestimated as compared with the treatment of [16]. Consequently, the behavior of $\Delta_{\max }\left(p_{F}\right)$ has a maximum value that exceeds by $2.5 \mathrm{MeV}$ the results shown in Fig. 2a of [16. However, this maximum value is reached for $p_{F} \sim 0.8 \mathrm{fm}^{-1}$ in both cases. The spreading is also similar, a drop of about $75 \%$ is verified at $p_{F} \sim 1.2 \mathrm{fm}^{-1}$ in both calculations.

As a last application we study the temperature behavior of the gap function evaluated at the Fermi momentum in the FEC approach. In fig. 4 we select 
some definite values of the particle density such that the gap has magnitude higher than $0.1 \mathrm{MeV}$ at zero temperature. For the lower densities a steep fall is registered around $T \sim 2.5 \mathrm{MeV}$.

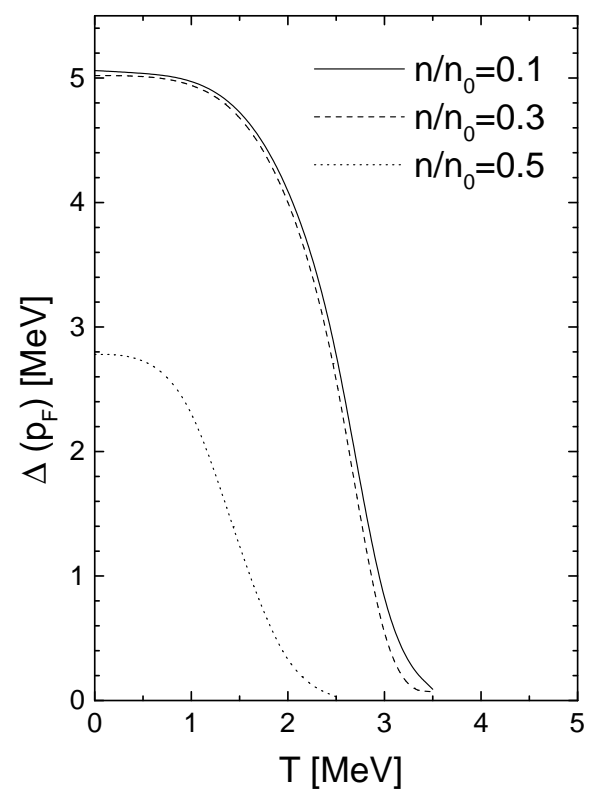

Figure 4: The gap energy for the ${ }^{1} S_{0}$ phase in terms of the temperature for several densities.

\section{Summary}

In this work we have studied the superfluid phase in a nuclear environment, within the relativistic field theory of hadrons. The Fermi-Landau liquid framework, as stated by [32, has been used in order to obtain expressions for the ${ }^{1} S_{0}$ superfluid gap energy and the distribution functions for both normal and superfluid phases. This is equivalent to a Hartree approximation, which provides a quasi-particle picture of nucleons dressed by the mesonic interaction, solved in a self-consistent way with a BCS scheme for the superfluid phase.

It is well known that BCS approaches in terms of QHD models, produce excessively large values for the gap in isospin symmetric nuclear matter. In this work we explored the possibility to state a easy to handle formalism, capable to retain the good properties of the QHD theory but modifying the above mentioned failure. With this purpose we took the simplest sigma-omega interaction and we introduced short range correlations inspired by the finite volume extension of nucleons. In our approach an additional model parameter is required, which 
is related to the characteristic length of the finite size of nucleons.

We have found a significative reduction, between $30-40 \%$, of the ${ }^{1} S_{0}$ gap in symmetric matter. So, the results obtained are closer to non-relativistic estimates. The modifications proposed modify the high momenta performance producing a pairing potential which goes to zero faster than in [9]. Further refinements, such as Fock term, additional mesons, non-linear sigma terms, etc., could improve the agreement with expected values.

Finally, the temperature behavior of the superfluid gap obtained is comparable with previous estimates [4, major differences correspond to the lowest densities.

\section{Acknowledgements}

This work was partially supported by the CONICET, Argentina.

\section{References}

[1] T. Tanigawa, M. Matsuzaki, and S. Chiba, Phys. Rev. C 70 (2004) 065801.

[2] M. Serra, A. Rummel, and P. Ring, Phys. Rev. C 65 (2001) 014304.

[3] W. Long, P. Ring, N. Van Giai, and J. Meng, Phys. Rev. C 81 (2010) 024308 .

[4] H. Muther and W.H. Dickhoff, Phys. Rev. C 72 (2005) 054313.

[5] W. Zuo, U. Lombardo, H. Schulze, and C.W. Shen, Phys. Rev. C 66 (2002) 037303 .

[6] S. Gandolfi, et al., Phys. Rev. Lett. 101 (2008) 132501.

[7] L.G. Cao, U. Lombardo, and P. Schuck, Phys. Rev. C 74 (2006) 064301.

[8] F. Matera, G. Fabbri, and A. Dellafiore, Phys. Rev. C 56 (1997) 228.

[9] H. Kucharek and P. Ring, Z. Phys. A 339 (1991) 23.

[10] J.-S. Chen, P.-F. Zhuang, and J.-L. Li,Phys. Lett. B 585 (2004) 85.

[11] J. Li, B. Y. Sun, and J. Meng, Int. J. Mod. Phys. E 17 (2008) 1441.

[12] M. Matsuzaki, Prog. Theor. Phys. 116 (2006) 127.

[13] D. J. Dean and M. Hjorth-Jensen, Rev. Mod. Phys. 75 (2003) 607.

[14] M. Matsuzaki, Phys. Rev. C 58 (1998) 3407

[15] M. Matsuzaki and T. Tanigawa, Phys. Lett. B 445 (1999) 254.

[16] M. Matsuzaki and T. Tanigawa, Nucl. Phys. A 683 (2001) 406. 
[17] B. D. Serot and J. D. Walecka, Advan. Nucl. Phys. 16 (1986) 1.

[18] B. D. Serot and J. D. Walecka, Int. J. Mod. Phys. E 6 (1997) 515.

[19] F. Hofmann, C. M. Keil, and H. Lenske, Phys. Rev. C 64 (2001) 034314, and references therein.

[20] D. H. Rischke, M. I. Gorenstein, H. Stöcker and W. Greiner, Z. Phys. C 51 (1991) 485.

[21] J. Cleymans and H. Satz, Z. Phys. C 57 (1993) 135.

[22] H. Kouno, K. Koide, T. Mitsumori, N. Noda, A. Hasegawa, M. Nakano, Prog. Theor. Phys. 96 (1996) 191.

[23] G. D. Yen, M. I. Gorenstein, W. Greiner, S. N. Yang, Phys. Rev. C 56 (1997) 2210.

[24] M. I. Gorenstein, H. Stöcker, G. D. Yen, S. N. Yang, W. Greiner, J. Phys. G 24 (1998) 1777.

[25] S. Kagiyama, A. Nakamura, T. Omodaka, Z. Phys. C 53 (1992) 163.

[26] S. Kagiyama, A. Nakamura, T. Omodaka, Z. Phys. C 56 (1992) 557.

[27] S. Kagiyama, A. Minaka, A. Nakamura, Prog. Theor. Phys. 89 (1993) 1227.

[28] C. P. Singh, B. K. Patra, K. K. Singh, Phys. Lett. B387 (1996), 680.

[29] R. Aguirre and A.L. De Paoli, LANL Report nucl-th/9907087

[30] P.K. Panda, M.E. Bracco, M. Chiapparini, E. Conte and G. Krein, Phys. Rev. C 65 (2002) 065206 .

[31] J. Kapusta, Phys. Rev. D 23 (1981) 2444.

[32] A.I. Akhiezer, V.V. Krasil'nikov, S.V. Peletminskii, and A.A. Yatsenko, Phys. Rep. 245 (1994) 1.

[33] I. Zahed and G. E. Brown, Phys. Rep. 142 (1986) 1.

[34] J. Rikovska-Stone, P. A. M. Guichon, H. H. Matevosyan, and A. W. Thomas, Nucl.Phys. A792(2007) 341.

[35] J. Zimanyi and S. A. Moszkowski, Phys. Rev. C 42 (1990) 1416. 